\title{
Environmental Pollution and Peripheral Artery Disease
}

\author{
Raffaele Serra $\mathbb{D}^{1-3}$ \\ Andrea Abramo ${ }^{1-3}$ \\ Nicola lelapi ${ }^{1,4}$ \\ Salvatore Procopio ${ }^{5}$ \\ Pietro Marino ${ }^{3}$ \\ 'Interuniversity Center of \\ Phlebolymphology (CIFL), International \\ Research and Educational Program in \\ Clinical and Experimental Biotechnology, \\ Department of Surgical and Medical \\ Sciences, University Magna Graecia of \\ Catanzaro, Catanzaro, Italy; ${ }^{2}$ Department \\ of Medical and Surgical Sciences, \\ University Magna Graecia of Catanzaro, \\ Catanzaro, Italy; ${ }^{3}$ Department of Social \\ Sciences, Vitambiente, Catanzaro, Italy; \\ ${ }^{4}$ Department of Public Health and \\ Infectious Disease, "Sapienza" University \\ of Rome, Rome, Italy; ${ }^{5}$ Department of \\ Catanzaro District, Laboratory of \\ Physics, Calabria Regional Agency for \\ Environment Protection, Catanzaro, Italy
}

Correspondence: Raffaele Serra

Interuniversity Center of

Phlebolymphology (CIFL), International

Research and Educational Program in

Clinical and Experimental Biotechnology,

Department of Surgical and Medical

Sciences, University Magna Graecia of

Catanzaro, Viale Europa, Località

Germaneto, Catanzaro, 88100, Italy

Tel +3909613647380

Email rserra@unicz.it

\begin{abstract}
Peripheral artery disease (PAD) of the lower limbs represents one of the most important clinical conditions among vascular disease and can negatively impact quality of life of affected patients, representing also an important socioeconomic burden. Several risk factors predispose to PAD and its complications. Nevertheless, the role of pollution in this context has not been fully evaluated and this article explored the most updated information on epidemiology and environmental pollution in order to hypothesize the possible contribution of air pollution in the onset of PAD. Pollution is an important problem for the global community and has harmful effects on human health and cardiovascular system, and, specifically, particulate matter $10\left(\mathrm{PM}_{10}\right)$ was found significantly associated with PAD.
\end{abstract}

Keywords: peripheral artery disease, pollution, environment, PM10, risk factors

\section{Introduction}

Vascular disease (VD) refers to a wide range of clinical conditions affecting arteries, veins and lymphatics. VD is an important cause of morbidity and mortality worldwide. ${ }^{1}$ Among VD, peripheral artery disease (PAD) of the lower limbs represents one of the most important clinical conditions of the vascular system. PAD is defined by an ankle-brachial index (ABI; the ratio of the systolic blood pressure at the ankle to the systolic blood pressure in the arm) of 0.90 or less. PAD is also generally linked to the risk of developing cardiovascular diseases in several cardiac and vascular districts, and therefore it can be considered a public health concern. PAD is related to atherosclerosis, and, in this context, is the third most frequent clinical event after ischemic heart disease and stroke. The range of symptoms of PAD affects significatively the quality of life of affected patients, as it varies from intermittent claudication to critical limb ischemia with the risk of minor or major amputation. ${ }^{1,2}$ Aging, smoking, diabetes, hypertension and hyperlipidaemia are considered important risk factors for PAD as well as for other atherosclerosis-related problems. ${ }^{2-4}$ Environmental pollution is also one of the most important cause of mortality and disability in the world today, and, somehow, it has also been associated with cardiovascular disease. Air pollution is a complex mixture of thousands of components. Among the most important constituents of this mixture we find: particulate matter (PM) classified in $\mathrm{PM}_{0.1}, \mathrm{PM}_{2.5}$ and $\mathrm{PM}_{10}$ and gaseous pollutants such as ozone (O3), nitrogen dioxide (NO2), benzopyrene (BaP), sulfur dioxide (SO2). ${ }^{5}$

As generally PAD still receives less attention compared to heart problems, ${ }^{2}$ the role of pollution in this context has not been evaluated yet, and the aim of this review 
article is to explore the most updated information on epidemiology and environmental pollution in order to hypothesize the possible contribution of air pollution in the onset of PAD.

\section{Materials and Methods}

We searched for epidemiological data of PAD and data regarding the concentrations of the main environmental pollutants. National Vitambiente Association and ArpaCal - Regional Agency for the Protection of the Environment of Calabria have contributed to this study by providing data included in pollutant databases and helping with processing of the collected data.

\section{Searching for Epidemiology of PAD}

We searched in PubMed (MEDLINE), Scopus, Science direct databases for articles published between Jan 1, 2011, and April 30, 2019; only the studies evaluating PAD prevalence in the general population were considered. Studies set in samples of hospitalized patients or with special features were excluded from this review because they were not considered representative of the general population.

\section{Searching for Environmental Pollution}

The environmental pollution data set was retrieved searching: Global Health Observatory (GHO) data repository, IndexMundi, United States Environmental Protection Agency (US EPA), IQair. Concentrations of environmental pollutants present in the air, in the urban environment, have been estimated through a set of indicators early drawn up annually with increasingly sophisticated methodologies and criteria, with two fundamental objectives: to investigate any correlations between the increase in pollutant concentration and the incidence of certain diseases; to assess the effectiveness of environmental policies applied by States and governments to contain air pollution, strongly correlated with the onset and development of such diseases.

The possible statistical correlations between some of the main pollutants and PAD in the various countries of the world were evaluated from 1990 to 2017. On the basis of annual trends in pollutant concentrations in the various countries, statistical correlation indicators were significant and satisfactory to our purposes.

\section{Results}

\section{Epidemiology of PAD}

The analysis of the collected epidemiological data revealed an overall prevalence of PAD in the world general population aged $\geq 25$ years is $5.56 \%$. In particular, the prevalence reached $7.37 \%$ in high-income countries and $5.09 \%$ in low- and middle-income countries. A total of 236 million people had PAD in 2015; the most affected age group was the one ranging between 45 and 49 years in the low- and middle-income countries (LMIC) (Table 1). ${ }^{2}$

PAD prevalence has been estimated in every country belonging to WHO; referring to American continent highincome countries (HIC), Chile has the highest prevalence of Pad with $8.07 \%$ while French Guiana has the lowest prevalence with $4.41 \%$ (Table 2$)^{2}$

Among LMIC of America, Cuba has the highest prevalence and Haiti the lowest one, respectively, with $6.88 \%$ and $4.23 \%$ (Table 3$)^{2}$

In the Eastern Mediterranean region, Lebanon reaches the first position (6.25\%); Remarkably, the prevalence of PAD in Qatar, which is among the lowest in the world, is $2.55 \%$ (Table 4) (Table 5). ${ }^{2}$

From the analysis of the PAD prevalence data of the Western Pacific region, Japan ranks first (7.79\%) followed by Micronesia $(7.47 \%)$. The prevalence in the states of this area spans from 3.3\% (Brunei Darussalam) to $7.79 \%$ of the aforementioned Japan (Tables 6 and 7).

In the African Region, the prevalence of PAD has a rather low trend with all values between $3-4 \%$, with only the Mauritius Islands and the Republic of Seychelles showing prevalence around $6 \%$ (Table 8 ).

The high-income countries of the European region have the largest number of patients affected by PAD.

Table I PAD Prevalence

\begin{tabular}{|l|l|l|l|}
\hline Age (Years) & HIC (\%) & LMIC (\%) & Worldwide (\%) \\
\hline $25-29$ & 1.72 & 2.91 & 2.75 \\
$30-34$ & 2.19 & 3.32 & 3.15 \\
$35-39$ & 2.79 & 3.79 & 3.62 \\
$40-44$ & 3.54 & 4.32 & 4.18 \\
$45-49$ & 4.50 & 4.93 & 4.85 \\
$50-54$ & 5.70 & 5.61 & 5.63 \\
$55-59$ & 7.21 & 6.38 & 6.57 \\
$60-64$ & 9.08 & 7.26 & 7.7 \\
$65-69$ & 11.36 & 8.24 & 9.16 \\
$70-74$ & 14.14 & 9.36 & 10.85 \\
$75-79$ & 17.43 & 10.62 & 12.92 \\
$80-84$ & 21.24 & 12.04 & 15.69 \\
$85-89$ & 25.52 & 13.66 & 19.28 \\
$>90$ years & 31.25 & 15.93 & 24.64 \\
Total (>25) & $7.37 \%$ & 5.08 & 5.56 \\
\hline
\end{tabular}

Abbreviations: HIC, high- income countries; LMIC, low-and middle- income countries. 
Table 2 PAD Prevalence in the America Region (HIC)

\begin{tabular}{|l|l|}
\hline Country & Prevalence of PAD (\%) \\
\hline Antigua and Barbuda & 5.06 \\
Argentina & 6.31 \\
Aruba & 5.83 \\
Bahamas & 4.58 \\
Barbados & 4.71 \\
Canada & 5.58 \\
Chile & 8.07 \\
Curaçao & 6.42 \\
French Guiana & 4.41 \\
Guadeloupe & 6.80 \\
Martinique & 6.88 \\
Puerto Rico & 6.14 \\
Trinidad and Tobago & 5.25 \\
United States of America & 5.84 \\
United States Virgin Islands & 6.56 \\
Uruguay & 7.10 \\
\hline
\end{tabular}

Table 3 PAD Prevalence in the America Region (LMIC)

\begin{tabular}{|l|l|}
\hline Country & Prevalence of PAD (\%) \\
\hline Belize & 4.08 \\
Bolivia & 4.99 \\
Brazil & 5.05 \\
Colombia & 4.54 \\
Costa Rica & 4.83 \\
Cuba & 6.88 \\
Dominica Republic & 4.8 \\
Ecuador & 4.29 \\
El Salvador & 4.57 \\
Grenada & 5.05 \\
Guatemala & 4.26 \\
Guyana & 4.75 \\
Haiti & 4.23 \\
Honduras & 4.37 \\
Jamaica & 5.4 \\
Mexico & 4.73 \\
Nicaragua & 4.35 \\
Panama & 4.44 \\
Paraguay & 4.8 \\
Peru & 4.36 \\
Saint Lucia & 5.73 \\
Saint Vincent and the Grenadines & 4.88 \\
Suriname & 5.26 \\
Venezuela & 5.02 \\
\hline
\end{tabular}

Greece has a prevalence of $12.47 \%$, followed by Croatia (12.05\%), and Latvia (11.29\%). Italy, for example, has a prevalence of $9.25 \%$, meaning almost one in ten individuals suffering from PAD (Table 9).
Table 4 PAD Prevalence in the Eastern Mediterranean Region (HIC)

\begin{tabular}{|l|l|}
\hline Country & Prevalence PAD (\%) \\
\hline Bahrain & 4.24 \\
Kuwait & 3.84 \\
Oman & 2.28 \\
Qatar & 2.26 \\
Saudi Arabia & 3.46 \\
State of Palestine & 3.72 \\
United Arab Emirates & 2.55 \\
\hline
\end{tabular}

Table 5 PAD Prevalence in the Eastern Mediterranean Region (LMIC)

\begin{tabular}{|l|l|}
\hline Country & Prevalence of PAD (\%) \\
\hline Afghanistan & 4.35 \\
Djibouti & 3.93 \\
Egypt & 5.35 \\
Iran & 4.05 \\
Iraq & 4.33 \\
Jordan & 5.66 \\
Lebanon & 6.25 \\
Libya & 4.95 \\
Moroda S.O & 5.38 \\
Pakistan & 4.96 \\
Somalia & 4.46 \\
Sudan & 4.59 \\
Syrian Arab Republic & 5.28 \\
Tunisia & 5.74 \\
Yemen & 4.03 \\
\hline
\end{tabular}

Table 6 PAD Prevalence in the Western Pacific Region (HIC)

\begin{tabular}{|l|l|}
\hline Country & Prevalence of PAD (\%) \\
\hline Australia & 4.3 \\
Brunei Darussalam & 3.3 \\
China, Macao & 4.87 \\
China, Taiwain & 5.76 \\
China, Hong Kong & 6.1 \\
Guam & 5.29 \\
Japan & 7.79 \\
New Caledonia & 5.36 \\
New Zealand & 4.91 \\
Republic of Korea & 6.22 \\
Singapore & 4.6 \\
\hline
\end{tabular}

The middle- and low-income countries of the European region have lower prevalence values compared to the highincome European countries; eg, Turkmenistan, where the PAD prevalence is $3.73 \%$ or Uzbekistan where PAD 
Table 7 PAD Prevalence in the Western Pacific Region (LMIC)

\begin{tabular}{|l|l|}
\hline Country & Prevalence of PAD (\%) \\
\hline Cambogia & 4.79 \\
China & 5.64 \\
Fiji & 5.65 \\
French Polynesia & 5.52 \\
Kiribati & 7.07 \\
Lao People's Democratic Republic & 5.17 \\
Malaysia & 5.11 \\
Micronesia & 7.47 \\
Mongolia & 5.15 \\
Papua New Guinea & 5.07 \\
Philippines & 4.96 \\
Samoa & 6.36 \\
Solomon Islands & 5.7 \\
Tonga & 6.42 \\
Vanuatu & 6.07 \\
Vietnam & 5.2 \\
\hline
\end{tabular}

patients count up to $3.93 \%$. Prevalence data are higher in Serbia (7.95\%) and Bulgaria (7.82\%) therefore being close to those of the high-income European region (Table 10, Table 11). ${ }^{2}$

\section{Environmental Pollution}

We also evaluated data on environmental pollution from 1990 to 2017, same period as for the epidemiological evaluation. The arithmetic mean was calculated in order to show a possible correlation with the PAD. Ozone (O3), benzopyrene $(\mathrm{BaP})$, sulphur dioxide $\left(\mathrm{SO}_{2}\right)$, nitrogen dioxide $\left(\mathrm{NO}_{2}\right)$, $\mathrm{PM}_{2.5}$ and $\mathrm{PM}_{10}$ were the pollutants studied (Table 11). ${ }^{2}$

\section{Correlation Between PAD Prevalence and Environmental Pollution}

The correlation between epidemiological data and environmental data has been evaluated by the $R_{2}$ index (Pearson's correlation coefficient), known also as the coefficient of determination, representing, in statistics, the possible relationship between two variables. An $R_{2}$ index $=1$ represents a strong dependence between the variables and indicates a direct correlation between them; a value of 0 means instead no dependency.

We observed a significant correlation between the $\mathrm{PM}_{10}$ and PAD, being the calculated R2 value around 0.5 , thus representing a minimum dependence between the two variables investigated (Figure 1). ${ }^{2}$ Instead, $\mathrm{R}_{2}$
Table 8 PAD Prevalence in the Africa Region (LMIC)

\begin{tabular}{|l|l|}
\hline Country & Prevalence of PAD (\%) \\
\hline Burundi & 4.02 \\
Comoros & 4.1 \\
Eritrea & 3.94 \\
Ethiopia & 3.73 \\
Kenya & 3.82 \\
Malawi & 4.05 \\
Mauritius & 5.89 \\
Seychelles & 5.65 \\
South Sudan & 4.1 \\
Uganda & 3.59 \\
Tanzania & 4.11 \\
Zambia & 3.91 \\
Zimbabwe & 3.97 \\
Angola & 4.02 \\
Cameroon & 4.28 \\
Central African Republic & 4.3 \\
Chad & 3.92 \\
Congo & 4.48 \\
Democratic Republic of the Congo & 4.01 \\
Guinea & 4.0 \\
Gabon & 4.25 \\
Sao Tome and Principe & 3.65 \\
Algeria & 4.56 \\
South Africa & 4.8 \\
Swaziland & 3.93 \\
Benin & 3.9 \\
Burkina Faso & 4.28 \\
Cabo Verde & 4.39 \\
Cote D'lvoire & 4.04 \\
Gambia & 4.04 \\
Guinea & 4.13 \\
Guinea - Bissau & 4.06 \\
Liberia & 4.14 \\
Mali & 4.35 \\
Niger & 4.02 \\
Nigeria & 3.68 \\
Senegal & 3.99 \\
Sierra Leone & \\
Togo & \\
\hline
\end{tabular}

index were 0.2 for PM 2.5 and benzopyrene (BaP) (Figures 2 and 3).

With regard to the other chemical parameters (data not shown) the values of the determination index were close to zero meaning, as expected, no dependence nor correlation.

Based on our findings, the correlation between $\mathrm{PM}_{10}$ and PAD is the most significant from a statistical point of view. 
Table 9 PAD Prevalence in the European Region (HIC)

\begin{tabular}{|l|l|}
\hline Country & Prevalence of PAD (\%) \\
\hline Austria & 10.88 \\
Belgium & 7.97 \\
Channel Islands & 9.32 \\
Croatia & 12.05 \\
Cyprus & 7.51 \\
Czechia & 10.16 \\
Denmark & 6.96 \\
Estonia & 10.6 \\
Finland & 8.01 \\
France & 9.79 \\
Germany & 10.65 \\
Greece & 12.47 \\
Hungary & 9.63 \\
Iceland & 5.98 \\
Ireland & 6.67 \\
Israel & 7.44 \\
Italy & 9.25 \\
Latvia & 11.29 \\
Lithuania & 10.05 \\
Luxembourg & 7.36 \\
Malta & 8.12 \\
Netherlands & 8.28 \\
Norway & 7.43 \\
Poland & 8.84 \\
Portugal & 8.26 \\
Slovakia & 7.96 \\
Slovenia & 8.02 \\
Spain & 9.46 \\
Sweden & 7.46 \\
Switzerland & 7.5 \\
United Kingdom & 6.94 \\
\hline
\end{tabular}

\section{Discussion}

Peripheral artery disease (PAD) is a chronic disease characterized by the gradual stenosis or occlusion of one or more artery of lower limbs, resulting in severe functional limitations, and also an increase in cardiovascular risk. ${ }^{2}$ PAD is considered a chronic disease that impact severely on socioeconomic systems also for the impairment of quality of life. ${ }^{6}$ PAD affects approximately over 236 million adults worldwide and has a prevalence between $2.26 \%$ (Qatar) and $12.47 \%$ (Greece). ${ }^{3}$ The presence of PAD increase with age, and the male/female ratio is generally reported as $2: 1$, probably due to hormonal differences. $^{7}$ The Framingham Offspring Study showed that the odds ratio of PAD was 2.6 for each 10 years of age. Black ethnicity is also an independent risk factor for PAD, with an odds ratio of $2 .{ }^{8}$
Table 10 PAD Prevalence in the European Region (LMIC)

\begin{tabular}{|l|l|}
\hline Country & Prevalence of PAD (\%) \\
\hline Kazakhstan & 5.42 \\
Kyrgyzstan & 4.76 \\
Tajikistan & 4.04 \\
Turkmenistan & 3.73 \\
Uzbekistan & 3.93 \\
Armenia & 5.87 \\
Azerbaijan & 4.87 \\
Georgia & 6.9 \\
Turkey & 5.25 \\
Belarus & 6.51 \\
Bulgaria & 7.82 \\
Republic of Moldova & 5.79 \\
Romania & 7.04 \\
Russian Federation & 7.31 \\
Ukraine & 6.81 \\
Albania & 6.29 \\
Bosnia and Herzegovina & 7.68 \\
Montenegro & 6.88 \\
Serbia & 7.95 \\
Tfyr Macedonia & 6.12 \\
\hline
\end{tabular}

Diabetes, smoking, dyslipidemia, hypertension, obesity and pollution are the most reliable risk factors for PAD, ${ }^{4}$ but the main cause of PAD is atherosclerosis, a systemic inflammatory process characterized by the formation of plaques consisting mainly of cholesterol that reduce blood flow in arterial vessels of the legs, especially during physical activity. Depending on its severity, PAD can be asymptomatic or symptomatic. Symptoms range from intermittent claudication, that is a cramping pain during walking, to critical limb ischemia with rest pain or even the formation of ulcers or gangrene, that can lead to the amputation of the affected limb. ${ }^{1,2}$ The outcomes for PAD patients after limb amputation are generally very poor, as approximately $30 \%$ ambulate with a prosthetic limb and $50 \%$ die within 2 years. Therefore, there financial, social, and psychological implications that highlight the need to develop strategies for the early identification of risk factors, prompt diagnosis, and adequate treatment of patients with PAD. ${ }^{7}$

All patients with PAD have an increased risk of stroke, myocardial infarction and thrombosis, so they need to change their lifestyle to reduce their cardiovascular risk profile. It is therefore important to change the main risk factors so quit smoking, reduce cholesterol, control 
Table I I Environmental Pollutant Concentrations

\begin{tabular}{|c|c|c|c|c|c|c|}
\hline Country & $\mathrm{PM}_{10}(\mu \mathrm{g} / \mathrm{m} 3)$ & $\mathrm{PM}_{2.5}(\mu \mathrm{g} / \mathrm{m} 3)$ & $\mathrm{O}_{3}(\mu \mathrm{g} / \mathrm{m} 3)$ & $\mathrm{NO}_{2}(\mu \mathrm{g} / \mathrm{m} 3)$ & $\mathrm{BaP}(\mathrm{ng} / \mathrm{m} 3)$ & $\mathrm{SO}_{2}(\mu \mathrm{g} / \mathrm{m} 3)$ \\
\hline Austria & 45.2 & 17.0 & 118.5 & 30.7 & 0.8 & 10.1 \\
\hline Belgium & 43 & 16.5 & 97.6 & 36.3 & 0.2 & 12.3 \\
\hline Bulgaria & 77.3 & 23.8 & 105.6 & 24.1 & 1.8 & 44.1 \\
\hline Croatia & 68.2 & 20.8 & 115.3 & 29.1 & 1.1 & - \\
\hline Cyprus & 69.0 & 17.0 & 111.0 & 30.0 & 0.3 & 7.1 \\
\hline Czechia & 50.8 & - & 110.2 & 25.0 & 1.8 & 25.7 \\
\hline Denmark & 40.3 & 11.0 & 93.0 & 27.8 & 0.2 & 5.9 \\
\hline Estonia & 26.5 & 8.0 & 96.4 & 14.8 & 0.3 & 5 \\
\hline Finland & 25.1 & 7.0 & 91.1 & 21.7 & 0.5 & 7.1 \\
\hline France & 40.8 & 15.3 & 111.7 & 27.2 & 0.3 & 11.8 \\
\hline Germany & 38.5 & 15.2 & 107.2 & 34.7 & 0.3 & 8.3 \\
\hline Greece & 53.8 & - & 103.7 & 37.8 & - & 17.7 \\
\hline Hungary & 49.5 & - & 117.7 & 29.5 & 1.6 & 19.5 \\
\hline Iceland & 25.6 & 10.9 & - & 10.0 & - & 4.4 \\
\hline Ireland & 28.6 & 10.7 & 88.9 & 24.6 & - & 17.6 \\
\hline Italy & 52.3 & 18.9 & 124.4 & 36.6 & 0.4 & 8.7 \\
\hline Latvia & 44.8 & 17.6 & 95.0 & 28.0 & 0.8 & 7.7 \\
\hline Lithuania & 48.6 & 21.7 & 86.4 & 21.4 & 1.6 & 5.8 \\
\hline Luxembourg & 36.4 & - & 95.9 & - & 0.3 & 7.8 \\
\hline Malta & 52.8 & 17.8 & 103.6 & 37.5 & 0.1 & 8.8 \\
\hline Netherlands & 37.4 & 14.6 & 95.3 & 31.1 & 0.1 & 10.6 \\
\hline Norway & 39.4 & 9.2 & 83.6 & 38.7 & - & 6.4 \\
\hline Poland & 59.2 & 24.9 & 106.0 & 22.8 & 4.2 & 29.6 \\
\hline Portugal & 38.2 & 9.8 & 109.7 & 25.9 & - & 4.3 \\
\hline Romania & 44.3 & 15.4 & 77.8 & 28.9 & - & 18.6 \\
\hline Slovakia & 49.9 & 18.8 & 122.9 & 29.6 & - & 14.9 \\
\hline Slovenia & 47.0 & 20.5 & 123.1 & 30.2 & 1.2 & 9.6 \\
\hline Spain & 32.2 & 10.5 & 108.2 & 26.4 & 0.1 & 13.3 \\
\hline Sweden & 42.4 & 6.1 & 90.5 & 27.7 & - & 3.9 \\
\hline Switzerland & 39.2 & - & 120.0 & 35.2 & 0.2 & 7.9 \\
\hline United Kingdom & 31.4 & 12.8 & 86.8 & 31.7 & 0.3 & 9.3 \\
\hline
\end{tabular}

Abbreviations: $\mathrm{PM}_{10}$, particulate matter ${ }_{10} ; \mathrm{PM}_{2.5}$, particulate matter $2.5 ; \mathrm{O}_{3}$, ozone; $\mathrm{NO}_{2}$, nitrogen dioxide; SO2, sulphur dioxide; Bap, benzopyrene.

hypertension and diabetes. ${ }^{9}$ The systemic distribution of atherosclerosis causes several serious clinical manifestations, and often PAD patients also suffer from clinically cerebral or coronary artery disease, which is reflected in the 6-fold increase in mortality from cardiovascular disease compared with non-PAD patients. ${ }^{7}$

Song et al performed an updated systematic review of population-based studies reporting the complete epidemiology of PAD worldwide in the general population at global, regional, and national levels. ${ }^{2}$ The study displays the prevalence of PAD in different geographical and income regions and in different countries and territories, and all the data shown was used in our study in order to match these data with the worldwide air pollution information that we retrieved from dedicated databases.
The correlation between environmental pollution and vascular diseases, particularly with $\mathrm{PAD}$, is becoming increasingly evident today. Pollution has harmful effects on human health and is an important problem for the global community. It is well known that air pollution is an important factor in the development and exacerbation of respiratory diseases such as asthma, and lung cancer and only recently the impact on cardiovascular diseases has been studied. ${ }^{5}$

Air pollution is a mixture of thousands of components. Among the most important constituents of this mixture are: atmospheric particulate matter (PM), gaseous pollutants such as ozone (O3), nitrogen dioxide (NO2) and volatile organic compounds such as benzopyrene, carbon monoxide (CO) and sulphur dioxide 


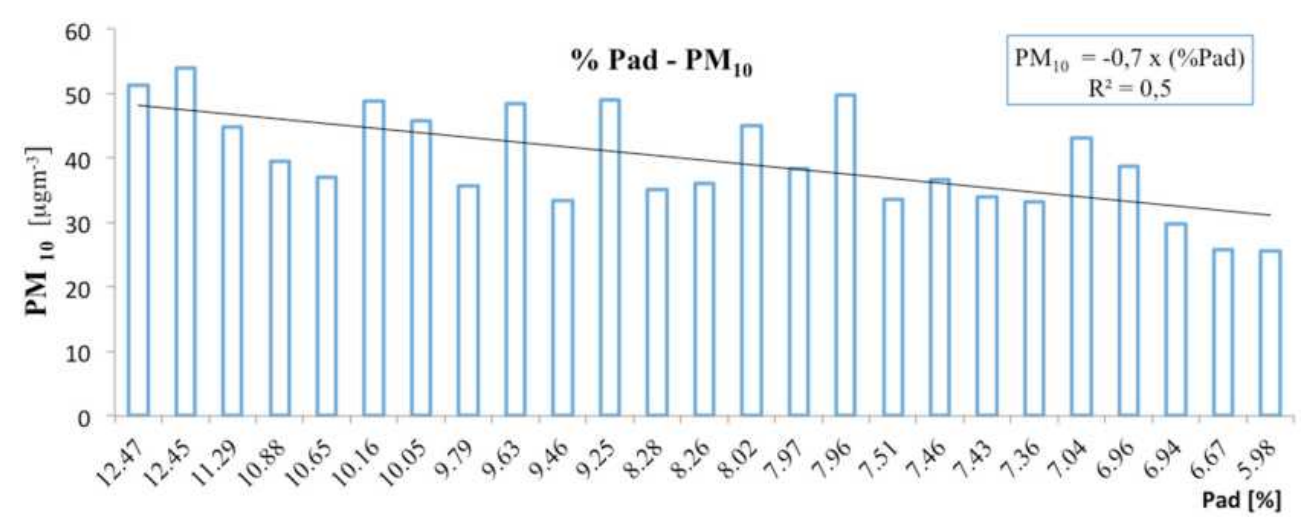

Figure I Correlation between $\mathrm{PM}_{10}$ and PAD.



Figure 2 Correlation between $\mathrm{PM}_{2.5}$ and PAD.



Figure 3 Correlation between $\mathrm{BaP}$ and PAD.

$\left(\mathrm{SO}_{2}\right)$. Primary pollutants, such as soot particles and nitrogen and sulphur oxides, are emitted directly into the air by burning fossil fuels. The main sources of $\mathrm{NO}_{2}$ are road traffic, electricity generation, industrial sources and residential heating. Secondary pollutants are formed by other components. An important example is ozone, which is formed through complex photochemical reactions of nitrogen oxides and volatile organic 
components. Particulate Matter (PM) is made up of particles from different sources and the particles are often classified into: coarse particles of diameter between 2.5 e $10 \mu \mathrm{m}$, fine particles of diameter less than $2.5 \mu \mathrm{m}$ and ultrafine particles $(<0.1 \mu \mathrm{m}){ }^{5}$

Environmental pollution determines the progression of existing cardiovascular diseases, but it also seems to have a role in the development of these diseases. The most relevant pathways that link air pollution to cardiovascular morbidity and mortality are induction of oxidative stress (OS), systemic inflammation, endothelial dysfunction, atherothrombosis and arrhythmogenesis. ${ }^{5}$

We focused on the possible correlation between air pollution and peripheral artery disease (PAD) of the lower limbs. Epidemiological research, matched to air pollution databases, has made it possible to exclude the link between certain environmental pollutants (NO2, SO2, O3) and PAD, and evidenced that the repeated and prolonged exposure over time to $\mathrm{PM}_{10}$ has a clear implication in the development and progression of PAD. Indeed, in our study, it has been observed that within countries where PAD prevalence is higher, there is a linear and progressive increase in $\mathrm{PM}_{10}$ values above the established and accepted minimum values.

Supporting our findings, some studies showed that $\mathrm{PM}_{10}$ determines the development of a systemic inflammatory response, including bone marrow stimulation, and in particular it is associated with the progression of the atherosclerotic process, that it is known to be the main cause of PAD. ${ }^{10}$

It is possible that $\mathrm{PM}_{10}$ is involved in the development of the atheroma, and therefore in the progression of PAD through the following mechanisms: $\mathrm{PM}_{10}$ inhalation can cause inflammation and OS within the lungs with mediators (eg, cytokines) which subsequently "flow into" the circulating system. Moreover, $\mathrm{PM}_{10}$ may enter directly into the bloodstream due to their small size reaching various organs such as adipose tissue. Adipose tissue is considered an important organ that releases adipokine including several hormones, growth factors, inflammatory cytokines. Long-term exposure to $\mathrm{PM}_{10}$ is believed to induce chronic inflammation in adipose tissue and also the release of adipokines into circulation. ${ }^{10}$

Blood pressure is a key factor in the development of atherosclerosis. It is known that inhalation of $\mathrm{PM}_{10}$ causes an increase in blood pressure through abnormal activation of the sympathetic nervous system. Increased blood pressure stimulates the expression of platelet-derived growth factor (PDGF) that is a potent mitogen for VSMCs and facilitates the formation of fibrous cap and the growth of atherosclerotic plaque till rupture and embolization. ${ }^{10}$

Oxidative stress is also crucial for the development of atherosclerosis. After exposure to $\mathrm{PM}_{10}$ there are high levels of reactive oxygen species (ROS) that can cause the development of atheroma, that progressively grows in size causing initial stenosis and then gradual occlusion of the affected artery, resulting in tissue ischemia. ${ }^{7}$ Exposure to air pollution may trigger several complex biological pathways involving, OS, various cell types and a wide range of molecular mediators including coagulation factors, pro-inflammatory cytokines such as tumor necrosis factor- $\alpha$ (TNF- $\alpha$ ), interleukin-6 (IL-6), growth factors such as vascular endothelial growth factor-A (VEGF-A) that could cause the alteration of the endothelial layer, that normally exerts a protective effect towards inflammation, cellular proliferation, and thrombosis. Therefore, endothelial dysfunction affects regular blood flow through arteries and predisposes to a pro-coagulant state with platelet aggregation and fibrinolytic impairment leading to atherogenesis. ${ }^{11}$ There are also several studies that link PM exposure to endothelial dysfunction mediated by OS. ${ }^{12-16}$ Moreover, OS, generated by PM pollution, is also related to the impairment of nitric oxide (NO) that, physiologically, controls vascular smooth muscle cells (VSMCs) that control relaxation of tunica media of the vessel wall. ${ }^{11}$ Reduced production and efficacy of NO in vessels is a hallmark of endothelial dysfunction. Several evidence in humans have shown vasoconstriction and altered flow-mediated dilation (FMD) using brachial ultrasound with PM exposure. PM seem also to exert negative effect on the vascular wall stimulating the angiotensin-1 receptor and uncoupling the endothelial NO synthase. It was also found that long-term exposure to PM might produce chronic changes in the brachial artery that negatively affects its ability to react to shear stress, primarily from NOmediated endothelial dysfunction. ${ }^{17}$ Other mechanisms mediated by air pollution are exerted on Endothelin-1 (ET-1) production, function and signaling and on Matrix Metalloproteinase - 9 (MMP-9) activities. ET-1 is a potent peptide vasoconstrictor that plays a significant role in regulating vascular homeostasis and is strictly associated with endothelium dysfunction including hypertension and atherosclerosis, while MMP-9 is a matrix in, a class of enzymes that belong to the zincmetalloproteinases family involved in the degradation of 
the extracellular matrix, and its role is pivotal in most vascular disease including PAD. ${ }^{18-20}$

A cross-sectional study in the Netherlands showed that air pollution exposure exerts adverse effects not only in adults but even in children as it determines important arterial wall changes and structure reducing also arterial distensibility in this age of life. ${ }^{21}$ More in general, assessing the arterial system, carotid intima media thickness (CIMT), carotid plaques and coronary artery calcification seem to be significantly PM related. ${ }^{22}$

Moreover, a study conducted in 19 European cities showed that a reduction in $\mathrm{PM}_{10}$ concentrations by only $5 \mu \mathrm{g} / \mathrm{m}^{3}$ can even prevent up to 7700 deaths per year for cardiovascular disease. ${ }^{23}$

The article by Chang et al illustrates the alterations of miRNA expression following exposure to different size and source of PM and their potential role in cardiovascular system, and these findings indicate that PM is able to even induce epigenetics changes determining important healthrelated effects. $^{24}$

All these effects, resulting from exposure to $\mathrm{PM}_{10}$, support the findings of this study, and in particular, that there is some correlation between PAD and air pollution. Nevertheless, we do not know how much the $\mathrm{PM}_{10}$ is involved in the development and progression of the PAD but we can postulate that it represents an important risk factor. It will be also important to evaluate at which exposure levels the association between $\mathrm{PM}_{10}$ and PAD may occur. Therefore, further large-scale studies are needed to confirm our hypothesis.

Control of exposure to air pollution and healthy style living may contribute to counteract the effects of air pollution. For example, the role of physical exercise, a lowpollution environment, in terms of cardiovascular health benefits are well established. Exercise can also attenuate the adverse effects of air pollution, as also showed in experimental animal studies. ${ }^{25}$ It is also well known that physical exercise has a positive effect on inflammation, immune cells and atherosclerosis improving walking distance in patients with PAD. ${ }^{26}$

Aerts et al examined the effect of residential green space (RGS) on general cardiovascular health. RGS are open pieces of land covered by vegetation such as forests, public parks, private gardens and urban street trees and they are able to attenuate environmental risk factors by means of avoiding air pollution, promoting physical activities, walking and social interactions. The study showed that these spaces were also associated with lower cardiovascular medication sales. $^{27}$

Other strategies to reduce the effects of air pollution on the cardiovascular system may be represented by the benefits of vitamins and nutrient supplementation and also by high-efficiency home air filtration systems that have been shown to reduce effectively indoor $\mathrm{PM}^{23}$

According to this background, prompt efforts to reduce exposure to air pollution should be urgently supported by appropriate and effective national pollution control plans. Health professionals have an important role to play in supporting educational and political initiatives, as well as in advising their patients. The reduction of exposure to air pollutants can be achieved also by general and simple measures of healthy living such as practicing physical activity in parks and gardens, avoiding the main roads or limit the time spent outdoors during periods of the day highly polluted.

The limit of this study, based on the heterogeneity of epidemiological studies retrieved, is the impossibility to portray multiple linear regression analysis with covariates. Therefore, larger studies with more robust statistical analysis are needed to confirm our findings.

\section{Funding}

This research received no specific grant from any funding agency in the public, commercial or not-for-profit sectors.

\section{Disclosure}

The authors reported no conflicts of interest for this work.

\section{References}

1. Ielapi N, Licastro N, Catana M, Bracale UM, Serra R. Vascular nursing and vascular surgery. Ann Vasc Surg. 2020;68:522-526. doi:10.1016/j.avsg.2020.05.038

2. Song P, Rudan D, Zhu Y, et al. Global, regional, and national prevalence and risk factors for peripheral artery disease in 2015: an updated systematic review and analysis. Lancet. 2019;7(8):e1020e1030.

3. Rajagopalan S, Al-Kindi SG, Brook RD, Pollution A. Cardiovascular disease: JACC state-of-the-art review. J Am Coll Cardiol. 2018;72 (17):2054-2070. doi:10.1016/j.jacc.2018.07.099

4. Shammas NW. Epidemiology, classification, and modifiable risk factors of peripheral arterial disease. Vasc Health Risk Manag. 2007;3 (2):229-234. doi:10.2147/vhrm.2007.3.2.229

5. Newby DE, Mannucci PM, Tell GS, et al; Group on Thrombosis, European Association for Cardiovascular Prevention and Rehabilitation; ESC Heart Failure Association. Expert position paper on air pollution and cardiovascular disease. Eur Heart J. 2015;36 (2):83-93b.

6. Brevetti G, Chiariello M. Peripheral arterial disease: the magnitude of the problem and its socioeconomic impact. Curr Drug Targets Cardiovasc Haematol Disord. 2004;4(3):199-208. doi:10.2174/ 1568006043336140 
7. Mascarenhas JV, Albayati MA, Shearman CP, Jude EB. Peripheral arterial disease. Endocrinol Metab Clin North Am. 2014;43 (1):149-166. doi:10.1016/j.ecl.2013.09.003

8. Selvin E, Erlinger TP. Prevalence of and risk factors for peripheral arterial disease in the United States: results from the National Health and Nutrition Examination Survey, 1999-2000. Circulation. 2004;110 (6):738-743. doi:10.1161/01.CIR.0000137913.26087.F0

9. Zemaitis MR, Boll JM, Dreyer MA. Peripheral arterial disease. In: StatPearls. Treasure Island (FL): StatPearls Publishing; 2020.

10. Bai Y, Sun Q. Fine particulate matter air pollution and atherosclerosis: mechanistic insights. Biochim Biophys Acta. 2016;1860 (12):2863-2868. doi:10.1016/j.bbagen.2016.04.030

11. Kelly FJ, Fussell JC. Role of oxidative stress in cardiovascular disease outcomes following exposure to ambient air pollution. Free Radic Biol Med. 2017;110:345-367. doi:10.1016/j. freeradbiomed.2017.06.019

12. Madrigano J, Baccarelli A, Wright RO, et al. Air pollution, obesity, genes and cellular adhesion molecules. Occup Environ Med. 2010;67 (5):312-317. doi:10.1136/oem.2009.046193

13. Bind MA, Coull B, Suh H, et al. A novel genetic score approach using instruments to investigate interactions between pathways and environment: application to air pollution. PLoS One. 2014;9(4): e96000.

14. Dai L, Bind MA, Koutrakis P, et al. Fine particles, genetic pathways, and markers of inflammation and endothelial dysfunction: analysis on particulate species and sources. J Expo Sci Environ Epidemiol. 2016;26(4):415-421. doi:10.1038/jes.2015.83

15. Wauters A, Dreyfuss C, Pochet S, et al. Acute exposure to diesel exhaust impairs nitric oxide-mediated endothelial vasomotor function by increasing endothelial oxidative stress. Hypertension. 2013;62 (2):352-358. doi:10.1161/HYPERTENSIONAHA.111.00991

16. Miller MR. Oxidative stress and the cardiovascular effects of air pollution. Free Radic Biol Med. 2020;151:69-87. doi:10.1016/j. freeradbiomed.2020.01.004

17. Krishnan RM, Adar SD, Szpiro AA. Vascular responses to long- and short-term exposure to fine particulate matter: MESA Air (Multi-Ethnic Study of Atherosclerosis and Air Pollution). $\mathrm{J} \mathrm{Am}$ Coll Cardiol. 2012;60(21):2158-2166. doi:10.1016/j. jacc.2012.08.973

Risk Management and Healthcare Policy

\section{Publish your work in this journal}

Risk Management and Healthcare Policy is an international, peerreviewed, open access journal focusing on all aspects of public health, policy, and preventative measures to promote good health and improve morbidity and mortality in the population. The journal welcomes submitted papers covering original research, basic science, clinical \& epidemiological studies, reviews and evaluations,
18. Finch J, Conklin DJ. Air Pollution-induced vascular dysfunction: potential role of Endothelin-1 (ET-1) system. Cardiovasc Toxicol. 2016;16(3):260-275. doi:10.1007/s12012-015-9334-y

19. Aflyatumova GN, Nigmatullina RR, Sadykova DI, Chibireva MD, Fugetto F, Serra R. Endothelin-1, nitric oxide, serotonin and high blood pressure in male adolescents. Vasc Health Risk Manag. 2018;14:213-223. doi:10.2147/VHRM.S170317

20. de Franciscis S, Serra R. Matrix metalloproteinases and endothelial dysfunction: the search for new prognostic markers and for new therapeutic targets for vascular wall imbalance. Thromb Res. 2015;136(1):5-6. doi:10.1016/j.thromres.2015.04.022

21. Ntarladima AM, Vaartjes I, Grobbee DE, et al. Relations between air pollution and vascular development in 5-year-old children: a cross-sectional study in the Netherlands. Environ Health. 2019;18 (1):50. doi:10.1186/s12940-019-0487-1

22. Hasslöf H, Molnár P, Andersson EM, et al. Long-term exposure to air pollution and atherosclerosis in the carotid arteries in the Malmö diet and cancer cohort. Environ Res. 2020;191:110095. doi:10.1016/j. envres.2020.110095

23. Medina S, Plasencia A, Ballester F, Mücke HG, Schwartz J; Apheis group. Apheis: public health impact of pm10 in 19 European cities. J Epidemiol Community Health. 2004;58(10):831-836.

24. Cheng M, Wang B, Yang M, et al. microRNAs expression in relation to particulate matter exposure: a systematic review. Environ Pollut. 2020;260:113961. doi:10.1016/j.envpol.2020.113961

25. Al-Kindi SG, Brook RD, Biswal S, Rajagopalan S. Environmental determinants of cardiovascular disease: lessons learned from air pollution. Nat Rev Cardiol. 2020;17(10):656-672. doi:10.1038/ s41569-020-0371-2

26. Dopheide JF, Geissler P, Rubrech J, et al. Influence of exercise training on proangiogenic TIE-2 monocytes and circulating angiogenic cells in patients with peripheral arterial disease. Clin Res Cardiol. 2016;105(8):666-676. doi:10.1007/s00392-016-0966-0

27. Aerts R, Stas M, Vanlessen N, et al. Residential green space and seasonal distress in a cohort of tree pollen allergy patients. Int J Hyg Environ Health. 2020;223(1):71-79. doi:10.1016/j.ijheh.2019.10.004 